\title{
Relevance of circulating tumor cells, extracellular nucleic acids, and exosomes in breast cancer
}

\author{
Anne M. Friel · Claire Corcoran · John Crown • \\ Lorraine O'Driscoll
}

Received: 22 December 2009/Accepted: 2 June 2010/Published online: 15 June 2010

(C) Springer Science+Business Media, LLC. 2010

\begin{abstract}
Early detection of cancer is vital to improved overall survival rates. At present, evidence is accumulating for the clinical value of detecting occult tumor cells in peripheral blood, plasma, and serum specimens from cancer patients. Both molecular and cellular approaches, which differ in sensitivity and specificity, have been used for such means. Circulating tumor cells and extracellular nucleic acids have been detected within blood, plasma, and sera of cancer patients. As the presence of malignant tumors are clinically determined and/or confirmed upon biopsy procurement-which in itself may have detrimental effects in terms of stimulating cancer progression/metastases-minimally invasive methods would be highly advantageous to the diagnosis and prognosis of breast cancer and the subsequent tailoring of targeted treatments for individuals, if reliable panels of biomarkers suitable for such an approach exist. Herein, we review the current advances made in the detection of such circulating tumor cells and nucleic acids, with particular emphasis on extracellular nucleic acids, specifically extracellular mRNAs and discuss their clinical relevance.
\end{abstract}

Keywords Breast cancer - Extracellular nucleic acids . Circulating tumor cells $\cdot$ Exosomes $\cdot$ Cancer stem cells

A. M. Friel · C. Corcoran · L. O’Driscoll ( $\square)$

School of Pharmacy and Pharmaceutical Sciences \& Molecular Therapeutics for Cancer Ireland, Trinity College Dublin, Dublin 2, Ireland

e-mail: lodrisc@tcd.ie

\section{J. Crown}

Molecular Therapeutics for Cancer Ireland, Dublin City University, c/o National Institute for Cellular Biotechnology Building, Glasnevin, Dublin 9, Ireland

\section{Introduction}

Breast cancer is the second most frequently diagnosed cancer after skin cancer in the US, with only lung cancer responsible for more female cancer-related deaths [1]. In recent times gene expression studies and immunohistochemical analyzes have classified breast cancer into five major subtypes depending on the presence or absence of hormone receptor expression and certain other markers.

Perou et al. [2] intrinsic classification defines these subtypes as basal-like breast cancers which largely correspond to estrogen receptor (ER) negative, progesterone receptor (PR) negative, and HER-2 negative tumors (i.e., triple negative); luminal A (mostly ER positive and lowgrade histologically); luminal B (also mostly ER positive, can express low levels of hormone receptors and often are high grade); HER-2 (also referred to as ErbB2) positive (show amplification and high expression of HER-2 gene); and triple negative tumors. Some basal-like cancers have high expression of cytokeratin (CK) 5, but as of yet, immunohistochemical methods of defining basal-like cancers are not well established due to logistical difficulties of performing many IHC markers in a clinical setting [3]. A final subtype is defined as normal breast-like tumors.

\section{Breast cancer recurrence}

The majority of breast cancer deaths are as a result of recurrent metastatic disease. Metastatic disease occurs as a result of a series of steps involving multiple host-tumor interactions [4] (reviewed by Gilbey et al. [5]). Primary tumor cells, whether they possess genetic mutations or have certain stem-like properties, grow aberrantly and may eventually detach, intravasate into the lymphatic and/or blood systems, before developing into secondary disease 
[4]. In breast cancer when tumor cells enter the lymphatic system, they travel to the sentinel nodes in the axilla and intercostal spaces before entering the bloodstream and subsequent progression to other organs [4]. In addition, tumor cells can disseminate directly through the blood to distant organs [6]. The detection and characterization of such cells would be an advantage in the identification of patients who are at risk of disease recurrence and the subsequent tailoring of individualized treatments.

The need and settings for breast cancer biomarkers

Breast cancer diagnosis is often merely confirmatory when a growth/lump is detected in the breast by the patient. Although mammography has greatly aided diagnosis for some patients (unfortunately it is dependent on mammography availability and also on the individual's age/breast tissue density, etc.), currently breast cancer is typically diagnosed on tissue biopsy. Individuals referred to a breast clinic (generally by their primary care physician) include those who will be diagnosed with breast cancer and those with a benign/harmless lump, e.g., fibroma. However, due to the lack of a blood/serum diagnostic test, biopsies must be assessed for all referred individuals. This often results in unnecessary delays for those with breast cancer, who need prioritising; unnecessary worry for those who have a benign condition; and an overall strain on clinics and health care systems. As outlined above, gene expression analysis of tumor tissue has enabled breast cancer sub-classification into five major sub-types. This information is relevant, as treatment options (e.g., hormone, Trastuzumab, chemotherapy) depends on the tumor sub-type. However, panels of biomarkers are urgently needed for earliest possible detection of breast cancer, for prognosing outcome and for predicting response to therapy, so as to determine those who require treatment versus over-treating those who do not, and to ensure that those who would benefit from neooadjuvant/adjuvant therapy receive the treatment to which they are most likely to respond. Undoubtedly, minimally invasive blood/serum tests that could aid in breast diagnosis (to triage individuals) and in tumor sub-typing (ensure optimal treatment selection) would contribute to timely, personalized management of those referred to the breast clinic; ultimately contributing to improved quality of life and overall survival.

No single biological biomarker can accurately detect breast cancer. The heterogeneity of breast cancer tumors is well documented. This is evident by the detection of tumor-initiating capabilities of certain cell populations, based on expression levels of specific surface markers $\left(\mathrm{CD} 44^{+}, \mathrm{CD}_{24}^{-/ \text {low }}, \mathrm{EpCAM}^{+}\right.$, Lineage $\left.^{-}\right)$[7]. These cells-often termed cancer stem cells, due to certain stemlike characteristics they share with normal stem cells-are capable of reconstituting tumors in vivo. Such cancer stem cells have also been documented in a variety of different tumors including ovary $\left(\mathrm{CD} 44^{+} / \mathrm{CD} 117^{+}\right)[8],\left(\mathrm{CD} 133^{+}\right)$ $[9,10]$, and endometrium [11].

\section{Circulating tumor cells}

Circulating tumor cells (CTCs) are defined as tumor cells circulating in the peripheral blood of patients, shed from either the primary tumor or from its metastases. The presence of solid tumor-derived cells in peripheral blood was first reported in 1869 [12]. CTC detection in breast cancer, based on expression of specific cell surface markers, is a fast emerging area of research with efforts focusing on detection of early metastatic disease. The development of a suitable assay has proven difficult due to the heterogeneous nature of CTCs. In general, there are two main methods for their detection. These are based on cytometric and nucleic acid manipulation. Both methods generally require an enrichment step to increase sensitivity of the assay. This step is based on either detection of specific surface markers using immuno-selection and/or on morphological features, such as cell size or density. Each of the several methods available to detect CTCs have distinct advantages and disadvantages. We present a brief description of the most clinically relevant assays used, including their associated advantages/disadvantages. Readers are directed towards a recent article for an in-depth review on technical and statistical considerations for CTC analyzes in the clinic [13].

Detection methods of circulating tumor cells

CellSearch $^{\circledR}$

The most established application of CTC analysis so far is based on CTC counts (CellSearch ${ }^{\circledR}$ System) and has been FDA approved for monitoring metastatic breast cancer [14]. This semi-automated technology combines both positive (epithelial cell adhesion molecule: EpCAM; using ferrofluid nanoparticles coated with anti-EpCAM antibodies) and negative (anti-CD45) selection. The enriched CTC population is then immunostained for specific markers and counted using a semi-automated fluorescence microscope, i.e., the CellSpotter analyzer (Veridex; Johnson \& Johnson). Thus, isolated CTCs are defined as $\mathrm{EpCAM}^{+}, \mathrm{CK}^{+}$, $\mathrm{DAPI}^{+}$, and $\mathrm{CD} 45^{-}$. In metastatic breast cancer an initial and first follow-up CTC count of $\geq 5$ per $7.5 \mathrm{ml}$ blood is predictive of shorter progression free survival and overall survival $[15,16]$. While a CTC count $\geq 5$ per $7.5 \mathrm{ml}$ blood at any time during therapy is indicative of a rapid disease progression and mortality [17]. A further study highlights 
the importance of use of the CellSearch ${ }^{\circledR}$ system as an independent prognostic factor for early breast cancer relapse [18]. However, at present (based on the most recent publication) the American Society of Clinical Oncology (ASCO) does not recommend the use of CTC detection for diagnosis or clinical management decisions in breast cancer [19]. Similarly even though the CellSearch ${ }^{\circledR}$ test is FDA approved, ASCO does not recommend its use for clinical decisions in metastatic breast cancer until further validation proves otherwise. While it is recognized that this method is highly sensitive, reproducible, commercially available, FDA approved, and has the capability of distinguishing between viable/non-viable cells, limitations include: usage of EpCAM to capture CTCs may miss some $\mathrm{EpCAM}^{-}$tumor cells, result in false positives (due to expression of the same antigens on non-tumor cells), the multiple enrichment/processing steps may also result in loss of CTC detection and no further processing of the CTC samples is possible.

\section{CTC-microchip}

Other immuno-selection approaches for CTC capture include the CTC-microchip. The CTC-chip is a microfluidics platform based on the interaction of target CTCs with antiEpCAM-coated microposts under precisely controlled laminar flow conditions, without requiring any pre-labeling or processing of specimens [20]. This gives the opportunity for further analysis of the captured, unfixed CTCs. Furthermore, good enrichment ( $98 \%$ cell viability) has been reported with this approach [21]. Limitations, similar to CellSearch ${ }^{\circledR}$, include the fact that only EpCAM-positive CTCs are captured in this way, the technology is not available commercially and clinical validation has yet to be achieved.

\section{EPISPOT}

An alternative antibody-based immunological approach is the Epithelial Immunospot (EPISPOT). It detects cells that secrete proteins such as cathespin-D, mucin-1 (MUC1) [22], and CK19; thus avoiding assessment of non-viable CTCs which would be unable to progress to form metastases [23]. However, as the CTCs are not captured per se, further analysis is not possible, isolated CTCs require $48 \mathrm{~h}$ culturing before analysis-delaying analysis and as for CTC-microchip—clinical validation has yet to be achieved.

\section{AdnaTest BreastCancer ${ }^{T M}$}

The application of AdnaTest BreastCancer ${ }^{\mathrm{TM}}$ test (AdnaGen AG) has generated interesting data thus far [24]. This "combination-of-combination" approach include immunoselection for cell surface markers including EpCAM and
MUC1 (AdnaGen BreastCancerSelect), followed by enhanced specificity through RT-PCR analysis for selected markers, including HER-2 and GA73.3-2 (AdnaGen BreastCancerDetect). Such assays have demonstrated that a change in the phenotype of tumor-derived cells can occur after initial diagnosis [21, 25]. Whilst the AdnaTest approach has been described as potentially having the same limitations (as for CellSearch ${ }^{\circledR}$ and CTC-Chip) of false positives and/or false negatives due to loss of antigens on CTCs, AdnaTest has also been proposed as suitable for characterizing events from stem cells to epithelial-mesenchymal transition (EMT) [26].

\section{Size and density approaches to CTC analyzes}

Size- or density-based approaches for CTC analysis include isolation by size of epithelial tumor (ISET) cells [27]. This filtering (through $8 \mu \mathrm{m}$-pore) approach is based on size differential between larger epithelial cells/CTCs and smaller leucocytes, followed by fixing and staining of the retained larger cells. Limitations of this approach, which deem it to be of limited sensitivity and specificity, include loss of small CTCs and retention of large leucocytes/ clumps of leucocytes. Density gradient approaches reported for CTC selection include OncoQuick ${ }^{\circledR}[28,29]$ and RossetteSep $^{\circledR}[30,31]$. In brief, the basic Ficoll-Hypaque differential density gradient centrifugation approach has been improved upon with OncoQuick ${ }^{\circledR}$ (includes special membranes to prevent cell cross-contamination) and RosetteSep ${ }^{\circledR}$ (includes an antibody cocktail which binds to unwanted hematopoietic cells, thus forming tetrameric complexes; which are subsequently avoided when selecting CTCs from the density gradient). Despite these advances on the basic density gradient approach, limitations described for OncoQuick ${ }^{\circledR}$ and RosetteSep $^{\circledR}$, respectively, include low specificity and possible cross-contamination of cells. As a comprehensive review of the application of these-and other approaches for CTC analysis is beyond the scope of this review, we suggest readers with a particular interest in CTC analysis in breast cancer see three reviews [26, 32, 33].

So far CellSearch ${ }^{\circledR}$ is the only standardized and validated test approved by the FDA. While this is likely to remain a very important approach to CTC analysis in breast cancer, studies from the Erasmus Medical Center, Rotterdam, indicate that not all subtypes of breast cancer (particularly normal-like breast tumor cells) are detectable by this method, emphasizing the need to increase the capabilities of this approach possibly by combining with some of the other technologies for CTC analysis outlined above. Furthermore, as CellSearch ${ }^{\circledR}$ only counts CTCs, undoubtedly combining this approach with additional technologies to capture more cells, different populations of cells, and the 
gene expression of cells, is necessary before CTC analysis is of more extensive use in the clinical setting. To date only one such multiparameter gene expression assay has gained approval by ASCO. The Oncotype DX ${ }^{\circledR}$ test, used on FFPE tumor tissue specimens, can be used to predict the risk of recurrence in patients treated with tamoxifen. The clinical usefulness of other multiparameter assays such as MammaPrint, "Rotterdam Signature" and the Breast Cancer Gene Expression Ratio are under investigation by ASCO.

\section{Characterization of circulating tumor cells}

Several studies have published on the characterization of CTCs and subsequent criteria used in the classification of CTCs [34]. Austrup et al., [35] reported on the prognostic significance of genomic alterations (HER-2 amplification) in CTCs derived from breast cancer patients. This study investigated amplification, loss of heterogeneity (LOH), and mutations. The presence and higher number of genomic imbalances in these CTCs were significantly associated with poorer prognosis. Genomic imbalances (present in CTCs from breast cancer patients) such as aneusomy are documented as originating from the primary tumor [36]. Meng et al. [37] demonstrated that CTCs can recapitulate the HER-2 status of the primary tumor. In a separate study the authors reported that a fraction of patients with HER-2 negative primary tumors had detectable HER-2 gene amplification in their CTCs, suggesting an acquired phenotype during cancer progression (9/24 patients; 37.5\%) [38].

So, while many of these formats of CTC analysis have great potential, it is likely that their routine incorporation into clinical laboratories is some way off and that their future application will be as a component of a panel of assays; rather than the definitive method.

\section{Disseminated tumor cells}

Minimal residual disease (presence of tumor cells not detectable by routine diagnostic procedures used in tumor staging after surgery) cannot be monitored by conventional clinical methods. Evidence suggests that tumor cells, of epithelial origin, can home to the bone marrow (BM). Such tumor cells found in the BM are named disseminated tumor cells (DTCs) (reviewed by Pantel et al. [39]). Several studies have assessed and reported on the prognostic values of DTCs in BM of breast cancer patients [40-42]. Braun et al., [41] analyzed data from nine clinical studies on the long term outcome of 4703 breast cancer patients presenting with and without DTCs. Strong evidence was presented for the adverse prognostic significance of initial BM DTC presence at the diagnosis of operable cancer. A similar study investigated BM DTCs from early stage breast cancer patients in recurrence free follow-up spanning a period of 29 months [42]. This study associated the positive presence of DTCs, at follow-up, with disease recurrence and highlighted the presence of persistent DTCs as being indicative of failed primary therapy, while suggesting that secondary adjuvant therapy may be of benefit in these patients. With regards to characterization of DTCs following therapy, HER-2 positive cells have been detected in breast cancer DTCs even though the corresponding primary tumor was HER-2 negative, implying that targeted therapy may be beneficial for such patients [43]. These studies provide compelling evidence of the importance of the initial detection and any subsequent detection and characterization following therapy of DTCs in BM of breast cancer patients. At present ASCO does not recommend the assessment of BM for DTC in the management of patients with breast cancer. In reality, the sampling of BM DTCs for monitoring purposes may not generally be feasible in a typical breast clinic and so DTC analysis is unlikely to become routine in the timeframe of some of the less-invasive analyzes (which involve more straightforward blood sampling) outlined in this review.

\section{Circulating nucleic acids and cancer}

Circulating nucleic acids are defined as extracellular nucleic acids (DNA or RNA) found circulating in the blood throughout the body. Such extracellular nucleic acids are thought to arise as a result of being secreted from cells; be it from normal cells, or malignant cells of primary or metastatic origin. The presence of circulating nucleic acids in plasma and serum has implications for minimally invasive diagnostic and predictive applications in benign and malignant conditions. Current terminology in literature for such nucleic acids include: circulating nucleic acids, cellfree nucleic acids and extracellular nucleic acids. For the purpose of this review we will refer to such nucleic acids as extracellular nucleic acids.

Evidence of extracellular nucleic acids in cancer

Extracellular nucleic acids, specifically extracellular DNA (exDNA), were first described in human plasma/serum by Mandel and Metais in 1948 [44]. Early studies reported increased levels of exDNA in sera from patients with lupus erythematosus [45, 46], rheumatoid arthritis [47], and glomerulonephritis [46]. Additionally, the presence of exDNA was reported in leukemia patients [46]. In 1977, with the discovery of elevated exDNA levels in patients with various cancers versus healthy individuals, the implications of extracellular nucleic acid detection for diagnostic purposes became evident [48]. 
Stroun et al. [49] first suggested that malignant tumors may be a source of exDNA, but it was only with the detection and verification of tumor-derived mutations of $\mathrm{K}$-ras in plasma from pancreatic adenocarcinoma patients [50] and point mutations of N-ras in plasma of patients with myelodysplastic syndrome and acute myelogenous leukemia [51] that an association between tumor and exDNA was convincing. Numerous studies since have reported on the presence of exDNA in sera/plasma from patients presenting with several different cancers, the most recent being studies on breast [52-54], lung [55, 56], and metastatic colon [57] cancers. Several authors have documented correlations between the methylation status of genes present in the primary tumor with that found in patient serum [58], mutation detection [57, 59, 60], and LOH [61]. Similarly the presence of exRNA is well documented in sera/plasma from patients presenting with breast [62-64] and colon cancers [65, 66].

\section{Origin of extracellular nucleic acids}

Questions remain as to the origin of extracellular nucleic acids, which can be found as free nucleic acids [67], bound to a cell's surface via proteins with specialized nucleic acid binding properties [68-70], complexed with proteolipids [71, 72] and within apoptotic bodies [73]. Studies have shown that they (DNA and RNA) can be released spontaneously from cells $[74,75]$. However, the exact mechanism of release from normal cells and tumor cells remains to be fully elucidated.

Anker et al. [76] and Jahr et al. [77] proposed tumor cell apoptosis/necrosis, apoptosis or lysis of CTCs, and/or active release from tumor cells as possible release mechanisms. RNA-proteolipid complexes/microvesicles/exosomes have been isolated from sera of cancer patients [78, 79] and from cancer cell lines [80, 81]. The importance of communication between tumor cells and their environment through fusion of shed membrane microvesicles to neighboring cells was discussed by Ratajczak et al. [82]. These microvesicles, 30-100 $\mathrm{nm}$ in diameter [83], can be shed from the cell surface or via inward budding of endosome membranes [84]. Once released into extracellular space these microvesicles are termed exosomes [85] and may be involved in trans-cellular signaling [86], transfer of membrane receptors, proteins, mRNA/microRNA [87], and organelles (e.g., mitochondria) between cells. Another role may be in the delivery of infectious and toxic agents (e.g., chemotherapeutic drugs) into cells [88] (reviewed by [82]).

It is thought the contents of exosomes depend on their cell of origin [78]; those derived from immune cells having immunostimulatory and anti-tumor effects in vivo [89], while those derived from tumor cells accelerate tumor growth [78, 90] and invasiveness [91, 92]. Additionally, the
RNA contained within exosomes is amplifiable; suggesting protection from RNase degradation by the exosome membrane [78]. Other groups have also demonstrated that serum/plasma RNAs in cancer patients are contained and protected in membrane bound structures [73, 93], (reviewed by [94]). Although circulating exosomes have been identified as having potential diagnostic relevance in some cancer types (including ovarian cancer [95]; glioblastomas [78]; and lung cancer [95]), the prevalence and clinical relevance of exosomes in sera from breast cancer patients has yet to be elucidated.

Extracellular RNAs and CTCs associated RNAs in breast cancer

More recent efforts have focused on the identification of possible biomarkers for diagnostic purposes in autoimmune and malignant diseases. In breast cancer exRNAs have been documented by a number of research groups [63, 9698]. We present the most documented studies on cell-free exRNAs and CTC associated RNAs.

\section{Cytokeratin 19}

Cytokeratins belong to a family of over 20 related polypeptides located in the cytoskeleton of various epithelial cells. Their main role is to provide cellular structure and integrity. They are classified into two types: acidic type I (CK9-CK20) and basic type II (CK1-CK8) [99]. Epithelial cells are targets of caspase-mediated proteolysis during apoptosis [100]. CK19 is cleaved by caspase 3 and soluble fragments (CYFRA 21-1), released into extracellular space, have been detected in sera of cancer patients $[62,101]$ and reportedly do not correlate with that of full-length CK19 mRNA [62].

Initially, Silva et al. [96] identified CK19 mRNA in plasma of breast cancer patients (49\%) in comparison to healthy controls $(20 \%)$. On evaluation of clinicopathological parameters (including patient age, tumor size, histopathological type and stage, lymph node metastases, ER and PR status, proliferative index) with molecular data, two parameters (tumor size and proliferative index as determined by Ki-67 IHC) were associated with the presence of plasma CK19 mRNA. As the recurrence rate in stage I and stage II breast cancer is between $10-20 \%$ and $40-65 \%$, respectively [102], it was proposed that the high proportion of patients in the study with early stage cancer (stage II) and showing positivity for CK19 mRNA expression, might be an indicator of patient clinical outcome and recurrence. Ninety percent of patients with detectable plasma CK19 mRNA were also positive for CK19-positive CTCs. Although the authors are unclear as to the significance of this finding, they suggest that shedding rates of nucleic 
acids from CTCs with metastatic potential may be higher than from tumor cells, i.e., that the exRNA identified in their study may have originated from CTCs.

More recent reports have demonstrated the prognostic significance of CK19 mRNA-positive CTCs in patients with early stage breast cancer [103-105]. Studies that investigated the prognostic value of CTCs/DTCs at the time of completion of adjuvant therapy [42, 106, 107] showed that the detection of DTCs [42, 108] and CTCs [106] after the completion of chemotherapy is associated with an unfavorable clinical outcome [103, 105]; further reviewed by Ignatiadis et al. [109]. In keeping with this work suggesting diagnostic and prognostic relevance for CK19, more recently full-length CK19 has been detected in bone marrow specimens from breast cancer patients and is associated with the presence of overt metastases and poor survival rates [110].

\section{Human telomerase reverse transcriptase}

Telomeres are a sequence of repetitive DNA located at the end of linear chromosomes where they serve to prevent the chromosome end from being recognized as DNA doublestranded breaks. Telomerase is the ribonucleoprotein reverse transcriptase complex that maintains telomere length and is reactivated in $90 \%$ of cancers [111]. It is composed of two subunits: an RNA component (TERC) and a telomerase reverse transcriptase (TERT). TERT is generally not expressed in most differentiated somatic cells; therefore, telomerase activity is absent. TERT is the major determinant for activation of telomerase. Absence of telomerase activity leads to a progressive shortening of telomeres and cellular senescence. However, reactivation of telomerase is considered a crucial step for malignant cells to become immortal.

Detection of hTERT expression either in plasma [65, 112], peripheral blood mononuclear cells [113] or by IHC in primary tumors [114] has been identified as diagnostic for prostate, colon, esophageal cancers, and paragangliomas, respectively. In terms of breast cancer, Chen et al. [97] identified hTERC and hTERT mRNA in primary tumors and sera of breast cancer patients presenting with invasive lobular carcinoma and invasive ductal carcinoma. Of the specimens analyzed, hTERT was identified in 25\% of sera and $94 \%$ of primary tumors, while hTERC was identified in $28 \%$ of sera and $94 \%$ of primary tumors. hTERC or hTERT was not detected in sera from control subjects or patients presenting with benign disease. No correlation was found between patient age, tumor size, tumor grade or the presence of nodal metastases.

A more recent study identified hTERT mRNA-positive CTCs [115]. Shen et al. [115] 2009 reported hTERT expression in $59.6 \%$ of patients presenting with breast cancer in comparison to healthy volunteers $(0 \%)$ and benign controls $(0 \%)$. Importantly, detection of hTERT mRNA significantly correlated with the TNM (internationally used tumor, node, metastases staging system) stage and lymph node metastasis, with hTERT present in 24.2, $67.9,80.0$, and $100 \%$ of patients with a TNM stage of I, II, III, and IV, respectively. In conjunction with hTERT, this group also investigated expression of survivin and hMAM (human mammaglobin), and reported improved sensitivity and specificity with all three biomarkers correlating to TNM stage. The authors conclude that the use of peripheral blood would be advantageous in clinical monitoring of early stage hematogenous spreading that may further develop into breast cancer metastasis or recurrence.

\section{Bmi-1}

Polycomb group (PcG) proteins are implicated in embryonic development and oncogenesis [116-118]. PcG protein expression is often dysregulated in several types of cancer and strongly correlates with an invasive and/or metastatic phenotype [119, 120]. Bmi-1 (PcG protein B lymphoma Mo-MLV insertion region 1 homolog), was initially identified as an oncogene cooperating with c-myc in the development of murine pre-B-cell lymphomas [121]. Bmi1 plays an essential role in maintaining the self-renewal of normal and malignant human mammary stem cells [122]. Over-expression of Bmi-1 is reported and correlated to poor clinical prognosis in solid tumors of breast [123], lung [124] and colon [125] cancers; to name but a few. Silva et al. [98] investigated Bmi-1 mRNA expression in plasma of a cohort of patients presenting with various subtypes and stages of breast cancer. Bmi-1 mRNA was detected in $43.2 \%$ of breast cancer specimens and also in $55 \%$ of healthy controls. However, expression levels were significantly higher in patients presenting with cancer versus the healthy controls. Upon correlation of gene expression with clinical-pathological features of the primary tumor (including vascular invasion, tumor subtype/stage, ER and PR receptor status, p53 status, HER-2 status, proliferative status), higher Bmi-1 levels were associated with p53positive immunostaining and PR-negative status. Importantly, the presence of plasma Bmi-1 mRNA was found to be associated with poorer survival at a more advanced clinical stage suggesting plasma Bmi-1 to be a potential surrogate biomarker of poor prognosis that could be analyzed minimally invasively.

\section{Human mammaglobin}

Human mammaglobin (MGB) belongs to the uteroglobin/ Clara cell protein family of small epithelial secretory proteins $[126,127]$ and was discovered by Watson and 
Fleming [128] in their isolation of sequence fragments that were abundantly expressed in breast tumors relative to normal breast tissue. The MGB1 promoter contains a polyoma enhancer-related motif which is associated with over-expression of HER-2 in breast cancer [129]. Two isoforms of MGB have been reported. These are mammaglobin 1 (also referred to as MGB1, mammaglobin A, SCG2A2) and mammaglobin 2 (MGB2, mammaglobin B, SCGB2A1). Most research on the use of MGB as a potential diagnostic biomarker in cancer has focused on MGB1, although there are reports of high levels of MBG2 mRNA and protein being associated with well (Grade 1) and moderately (Grade 2) differentiated endometrioid endometrial tumors [130].

Initially, Watson and Fleming [131] detected MGB1 expression in several breast carcinoma cell lines but not in primary breast stromal cells nor in immortalized luminal ductal breast cell lines. They concluded that MGB1 expression is mammary-specific, as MGB1 was expressed exclusively in mammary epithelial cells. It was since reported, however, that MGB1 is expressed at the mRNA and protein level in tumors of the endometrium, ovary, and cervix [132]. Watson and Fleming [131] also reported that primary breast carcinomas over-expressed MGB1 relative to normal breast tissue specimens, with approximately $50 \%$ of breast carcinoma cell lines and metastatic breast tumors analyzed exhibiting high levels of MGB1 mRNA. This over-expression did not appear to correlate with histology, tumor grade, tumor stage, or ER/PR status. A more recent study reported high MGB1 levels in primary breast cancers to be indicative of a less aggressive tumor phenotype and correlating with the expression of ER and PR, low Ki-67 labeling and absence of nodal invasion [133]. There has, so far, been no reported documentation of circulating transcripts of MGB1, however, MGB1 protein has been detected in sera of breast cancer patients [134, 135].

Recent efforts have focused on the detection of MGB1 in peripheral blood [136-138] as a diagnostic biomarker of metastatic disease. Mikhitarian et al. [139] used a panel of biomarkers (CK19, MUC1, MGB1, EpCAM, HER-2, prolactin inducible protein) in their investigation of a possible correlation between mRNA expression in peripheral blood CTCs, bone marrow DTCs and clinicopathological indicators (tumor type, TNM, ER/PR status, HER-2 status, age, and race). Their main finding was the lack of correlation between biomarker positivity in CTCs and tumor type and/or biomarker status of axillary lymph nodes. However, a significant association was reported between biomarker positivity in CTCs and tumor grade (grade II-III versus grade I), with this association holding true for expression of MGB1 mRNA alone. As this was an interim report of an ongoing study, the clinical significance in terms of overall survival and disease-free survival remain to be seen.

\section{Cyclin D1}

Cyclin D1 is a cell cycle regulator. It is induced by a variety of factors, such as epithelial growth factor, hormones and oncogenic signals and its over-expression is one of the most commonly observed alterations in cancer [140]. In breast cancers, cyclin D1 is over-expressed by $30-50 \%$ of tumors [140] and is documented as being predictive of poor clinical outcome [141]. García et al. [63] investigated whether plasma mRNA from breast cancer patients is related to disease-free survival and overall survival. Presence of cyclin D1 plasma mRNA was significantly associated with non-responsive patients following treatment after relapse. Additionally the authors reported a trend towards a significant relation between reduced overall survival rates and cyclin D1 presence. No association was detected between cyclin D1 plasma mRNA presence and disease-free survival.

\section{HER-2}

Approximately $25 \%$ of breast cancers over-express HER-2. Trastuzumab, a monoclonal antibody against the HER-2 receptor is the only approved targeted agent for treatment of HER-2 over-expressing cancers. However, not all patients respond to treatment and some develop resistance. The most frequent approach to measure HER-2 levels is by FISH, IHC, and ELISA. The extracellular domain of HER-2 can be proteolytically cleaved from the cell membrane and this domain can be measured in peripheral blood. Patients with HER-2 positive breast cancer have high serum HER-2 levels, which correlate positively with the number of metastatic sites and are indicative of disease recurrence [142]. While the presence and thus relevance of HER-2 mRNA in serum or plasma has yet to be investigated, detection of HER-2 mRNA-positive CTCs in blood specimens from patients with operable breast cancer have been reported as prognostic of unfavorable outcome, in terms of both relapse-free survival and overall survival [143].

As detailed above, research to date indicates that mRNAs (including CK19, TERT, Bmi-1, MGB, cyclin D1, and HER-2, to name but a few) exist in the systemic circulation-either freely or sequestered in microvesicles or exosomes. Undoubtedly, such mRNAs have potential as breast cancer biomarkers. However, while these studies have "proven-the-principle" and opened up an opportunity for biomarker searches that was initially considered to be irrelevant due to the high levels of RNase enzymes in this environment, we believe that more extensive studies are now needed to identify and validate the most relevant panels of mRNAs in large cohorts of relevant cases. 


\section{MicroRNAs in breast cancer}

MicroRNAs (miRNAs) are a family of endogenous small noncoding RNA molecules, of 19-28 nucleotides in length (reviewed: [144-146]). Over the past 2 years, since the first report of circulating microRNAs (miRNAs) associated with diffuse large B-cell lymphoma [147], circulating miRNAs have been reported in studies of a range of other cancer types. Specifically, in prostate cancer, serum $m i R$ 141 level was found to distinguish metastatic prostate patients $(n=25)$ from age-matched controls [148]. Similarly, $m i R-25$ and $m i R-223$ were reported as non-small cell lung cancer specific following their analysis in sera from 152 patients compared to 75 healthy volunteers [149]. Circulating miRNAs, albeit in exosomes, have also been reported to have potential as biomarkers for ovarian cancer [150] and for glioblastoma [78]. Studies of circulating miRNAs associated with breast cancer have been limited to date. In our pilot study, in addition to circulating mRNAs [64], we detected circulating miRNAs (including $m i R-141$ and $m i R N A-195)$ to be present at significantly higher levels $(P<0.001)$ in serum from patients recently diagnosed with breast cancer compared to age- and gender-matched healthy volunteers (as yet unpublished). In agreement with our findings, mi-195 and let-7a have recently been reported as at significantly higher levels in blood from breast cancer patients, compared to healthy volunteers [151].

Therefore, while studies of circulating miRNAs are still in their infancy, analyzes performed thus far suggested that these miRNAs - whether freely existing or enclosed in exosomes/microvesicles-may prove to have great potential as biomarkers for many cancers, including breast cancer. Due to their short sequences and stability (e.g., can be routinely assessed in formalin-fixed paraffin-embedded tissue and apparently can withstand many rounds of freezethawing when in solution) it is possible that miRNAs may have certain advantages over mRNAs as circulating biomarkers. Alternatively, a biomarker panel could conceivably include single or multiplex co-analysis of both types of RNA molecules; possibly offering some advantages over protein biomarker analysis. Now that their potential usefulness in this setting has been established, more extensive studies are essential in order to establish how best to exploit our understanding of circulating RNA molecules in the interest of breast cancer patients.

\section{Limitations of blood-based biomarkers and future directions}

Although research into potential cancer biomarkers has been ongoing for many years, the actual number of markers used in clinical settings remains limited [152-154]. The main reason being the discovery and development of useful biomarkers pose many challenges, with several factors contributing to the slow rate of biomarker development $[19,155]$. The ASCO published a report on recommendations for the use of tumor markers in breast cancers. They report a lack of validation of potential biomarkers as being a major obstacle in the development of new biologics or treatment strategies for patients. Areas that contribute to this problem include non-uniform procurement, processing and storage of specimens, differences in technologies used, non-standardized assays/non-validated equipment-all contributing to lack of study reproducibility, inappropriate statistical analyzes and insufficient patient specimen numbers. In addition, while some studies may show promising results, the overall lack of sufficient high-quality studies coupled with a lack of consistent data results in their omission from use as clinical markers for breast cancers [19].

To date, ASCO has recommended eight tumor biomarkers for breast cancer-all of which are proteins, i.e., CA 15-3 and CA 27.29, carcinoembryonic antigen (CEA), ER, PR, HER-2, urokinase plasminogen activator (uPA), and plasminogen activator inhibitor (PAI)-1. CA 15-3, CA 27.29, and CEA are biomarkers for monitoring; ER, PR and HER-2 are biomarkers for treatment planning; and UPA and PAI-1 are biomarkers for recurrent risk prediction [19]. While these are the only markers ASCO approved for clinical use, ASCO does not discourage the use of other novel or innovative approaches in the context of clinical trials (e.g., I-SPY 2 trial [156]). For future advancement in clinically useful biomarkers, well-designed, collaborative studies involving the inclusion of specimens-procured, processed, and analyzed using standard operating procedures-from large cohorts of consenting patients and healthy volunteers (for example, in Ireland such studies are possible, as facilitated by the All Ireland Irish Clinical Oncology Research Group (ICORG), as well as through international collaborations) should help in identifying and validating biomarkers panels which can be translated to the clinic, in the interest of cancer patients.

In 2001, Pepe et al. [157] defined five phases of biomarker development for early detection of cancer, i.e., (1) preclinical exploratory studies; (2) clinical assay development; (3) retrospective longitudinal repository studies; (4) prospective screening studies; and (5) cancer control studies assessing impact of screening on reducing the burden of disease. While all breast cancer biomarker studies are not aimed at diagnosis, the same overall strategy is very relevant to consider. An open mind should be kept as to what the final portfolio of any given biomarker panel may include. So far, there are no proven advantages of one molecule type over another with regards to their potential as biomarkers. Although, 
historically, focus has mainly been on proteins and more recently CTCs, results emerging from exploratory studies suggest that RNAs - which are proving to be reproducibly detected and can be amplified using a multiplex approach (thus minimizing the amount of specimen required for analysis) will form members of useful breast cancer biomarker panels. To date, what could possibly be best described as proof-of-concept studies have been reported on RNAs (mRNAs and miRNAs) as biomarkers. Relative to protein research, however, we believe that this field of research is still in its infancy and should now be much more extensively explored. While this has yet to be established, it is probable that optimal sensitivity and specificity will include a combination of mRNA, miRNA, protein-and possibly also CTC-analysis from a patient's blood specimen. It is our view that it is only through carefully designed research efforts and systems that are patient-orientated and collaborative, that the development of robust panels of such biomarkers and associated reliable assays will translate to the clinic to contribute to improvements in personalized treatment, quality of life and survival for breast cancer patients.

\section{Conclusion}

Breast cancer is the second leading cause of cancer-related deaths in the US and the most common cancer in women in the western world. The need for rapid methods for early detection of breast cancer, prognosing outcome, and predicting response to treatment-by analyzing CTCs or specific circulating protein and/or nucleic acids-would greatly aid current treatment modalities. Additionally, such biomarkers could potentially be used for continual monitoring of tumor response to treatment allowing for a change in treatment regime if needed, i.e., tailoring treatment for the individual.

In the past, the development of suitable assays to detect cancer cells has proven difficult due to the heterogeneous nature of breast cancer tumors. Current advances in molecular profiling have enabled certain surface biomarkers and gene expression patterns to be used for identification of breast tumor cells and their associated genes. As the majority of breast cancer deaths are as a result of recurrent metastatic disease, which may be caused by spread of CTCs/DTCs resulting from tumor resection, a minimally invasive method for cancer detection and monitoring would be clinically highly advantageous. In addition such a system has the potential to allow for early detection screening of people at increased risk of cancer. For the most part, biomarkers are not yet ready for routine use due to challenges in their clinical validation for breast cancer detection/diagnosis, prognosis and response to treatment prediction. However, advancing on the recent exciting studies reviewed here, current and future research focussing on the identification of diagnostic, prognostic and/or predictive circulating nucleic acids/CTCs has immense importance clinically to the development and improvement of current diagnostic and therapeutic options for breast cancer patients.

Acknowledgments The authors wish to thank the Science Foundation Ireland, Strategic Research Cluster award to Molecular Therapeutics for Cancer Ireland (award 08/SRC/B1410) for funding associated with preparation of this review.

\section{References}

1. Jemal A et al (2008) Cancer statistics, 2008. CA Cancer J Clin 58(2):71-96

2. Perou $C$ et al (2000) Molecular portraits of human breast tumours. Nature 406(6797):747-752

3. Sotiriou C, Pusztai L (2009) Gene-expression signatures in breast cancer. N Engl J Med 360(8):790-800

4. Jiang WG et al (2002) Molecular detection of micro-metastasis in breast cancer. Crit Rev Oncol Hematol 43(1):13-31

5. Gilbey AM et al (2004) The detection of circulating breast cancer cells in blood. J Clin Pathol 57(9):903-911

6. Pantel K, Brakenhoff R (2004) Dissecting the metastatic cascade. Nat Rev Cancer 4(6):448-456

7. Al-Hajj M et al (2003) Prospective identification of tumorigenic breast cancer cells. Proc Natl Acad Sci USA 100(7):3983-3988

8. Zhang $S$ et al (2008) Identification and characterization of ovarian cancer-initiating cells from primary human tumors. Cancer Res 68(11):4311-4320

9. Baba $T$ et al (2009) Epigenetic regulation of CD133 and tumorigenicity of CD133+ ovarian cancer cells. Oncogene 28(2):209-218

10. Curley M et al (2009) CD133 Expression defines a tumor initiating cell population in primary human ovarian cancer. Stem Cells 27(12):2875-2883

11. Rutella $S$ et al (2009) Cells with characteristics of cancer stem/ progenitor cells express the CD133 antigen in human endometrial tumors. Clin Cancer Res 15(13):4299-4311

12. Ashworth T (1869) A case of cancer in which cells similar to those in the tumours were seen in the blood after death. Aust Med J 14:146-147

13. Allan AL, Keeney M (2010) Circulating tumor cell analysis: technical and statistical considerations for application to the clinic. J Oncol 2010:426218

14. Liu M et al (2009) Circulating tumor cells: a useful predictor of treatment efficacy in metastatic breast cancer. J Clin Oncol 27(31):5153-5159

15. Cristofanilli $M$ et al (2004) Circulating tumor cells, disease progression, and survival in metastatic breast cancer. N Engl J Med 351(8):781-791

16. Budd $\mathrm{G}$ et al (2006) Circulating tumor cells versus imagingpredicting overall survival in metastatic breast cancer. Clin Cancer Res 12(21):6403-6409

17. Hayes D et al (2006) Circulating tumor cells at each follow-up time point during therapy of metastatic breast cancer patients predict progression-free and overall survival. Clin Cancer Res 12(141):4218-4224

18. Pierga $J$ et al (2008) Circulating tumor cell detection predicts early metastatic relapse after neoadjuvant chemotherapy in large 
operable and locally advanced breast cancer in a phase II randomized trial. Clin Cancer Res 14(21):7004-7010

19. Harris L et al (2007) American Society of Clinical Oncology 2007 update of recommendations for the use of tumor markers in breast cancer. J Clin Oncol 25(33):5287-5312

20. Nagrath $S$ et al (2007) Isolation of rare circulating tumour cells in cancer patients by microchip technology. Nature 450(7173): 1235-1239

21. Tewes $\mathrm{M}$ et al (2009) Molecular profiling and predictive value of circulating tumor cells in patients with metastatic breast cancer: an option for monitoring response to breast cancer related therapies. Breast Cancer Res Treat 115(3):581-590

22. Alix-Panabières $\mathrm{C}$ et al (2005) Characterization and enumeration of cells secreting tumor markers in the peripheral blood of breast cancer patients. J Immunol Methods 299(1-2): $177-188$

23. Alix-Panabières C et al (2007) Detection and characterization of putative metastatic precursor cells in cancer patients. Clin Chem 53(3):537-539

24. Fehm $\mathrm{T}$ et al (2009) Detection and characterization of circulating tumor cells in blood of primary breast cancer patients by RT-PCR and comparison to status of bone marrow disseminated cells. Breast Cancer Res 11(4):R59

25. Fehm T et al (2007) Determination of HER2 status using both serum HER2 levels and circulating tumor cells in patients with recurrent breast cancer whose primary tumor was HER2 negative or of unknown HER2 status. Breast Cancer Res 9(5):R74

26. Alunni-Fabbroni M, Sandri M (2010) Circulating tumour cells in clinical practice: methods of detection and possible characterization. Methods 50(4):289-297

27. Vona $\mathrm{G}$ et al (2000) Isolation by size of epithelial tumor cells: a new method for the immunomorphological and molecular characterization of circulating tumor cells. Am J Pathol 156(1): $57-63$

28. Gertler R et al (2003) Detection of circulating tumor cells in blood using an optimized density gradient centrifugation. Recent Results Cancer Res 162:149-155

29. Müller V et al (2005) Circulating tumor cells in breast cancer: correlation to bone marrow micrometastases, heterogeneous response to systemic therapy and low proliferative activity. Clin Cancer Res 11(10):3678-3685

30. Naume B et al (2004) Detection of isolated tumor cells in peripheral blood and in BM: evaluation of a new enrichment method. Cytotherapy 6(3):244-252

31. Hayes $\mathrm{G}$ et al (2010) Isolation of malignant $\mathrm{B}$ cells from patients with chronic lymphocytic leukemia (CLL) for analysis of cell proliferation: validation of a simplified method suitable for multi-center clinical studies. Leuk Res 34(6):809-815

32. Mostert B et al (2009) Circulating tumor cells (CTCs): detection methods and their clinical relevance in breast cancer. Cancer Treat Rev 35(5):463-474

33. Cristofanilli M, Braun S (2010) Circulating tumor cells revisited. JAMA 303(11):1092-1093

34. Fehm T et al (2005) Methods for isolating circulating epithelial cells and criteria for their classification as carcinoma cells. Cytotherapy 7(2):171-185

35. Austrup F et al (2000) Prognostic value of genomic alterations in minimal residual cancer cells purified from the blood of breast cancer patients. Br J Cancer 83(12):1664-1673

36. Fehm T et al (2002) Cytogenetic evidence that circulating epithelial cells in patients with carcinoma are malignant. Clin Cancer Res 8(7):2073-2084

37. Meng S et al (2006) uPAR and HER-2 gene status in individual breast cancer cells from blood and tissues. Proc Natl Acad Sci USA 103(46): 17361-17365
38. Meng S et al (2004) HER-2 gene amplification can be acquired as breast cancer progresses. Proc Natl Acad Sci USA 101(25): 9393-9398

39. Pantel K et al (2008) Detection, clinical relevance and specific biological properties of disseminating tumour cells. Nat Rev Cancer 8(5):329-340

40. Braun $S$ et al (2009) The prognostic impact of bone marrow micrometastases in women with breast cancer. Cancer Invest 27(6):598-603

41. Braun $S$ et al (2005) A pooled analysis of bone marrow micrometastasis in breast cancer. N Engl J Med 353(8):793-802

42. Janni W et al (2005) The persistence of isolated tumor cells in bone marrow from patients with breast carcinoma predicts an increased risk for recurrence. Cancer 103(5):884-891

43. Krawczyk N et al (2009) HER2 status on persistent disseminated tumor cells after adjuvant therapy may differ from initial HER2 status on primary tumor. Anticancer Res 29(10):4019-4024

44. Mandel P, Metais P (1948) Les acides nucleiques du plasma sanguine chez l'homme (in French). C R Seances Soc Biol Fil 142(3-4):241-243

45. Tan EM et al (1966) Deoxybonucleic acid (DNA) and antibodies to DNA in the serum of patients with systemic lupus erythematosus. J Clin Invest 45(11):1732-1740

46. Koffler D et al (1973) The occurrence of single-stranded DNA in the serum of patients with systemic lupus erythematosus and other diseases. J Clin Invest 52(1):198-204

47. Leon S et al (1977) Free DNA in the serum of rheumatoid arthritis patients. J Rheumatol 4(2):139-143

48. Leon S et al (1977) Free DNA in the serum of cancer patients and the effect of therapy. Cancer Res 37(3):646-650

49. Stroun M et al (1989) Neoplastic characteristics of the DNA found in the plasma of cancer patients. Oncology 46(5):318-322

50. Sorenson G et al (1994) Soluble normal and mutated DNA sequences from single-copy genes in human blood. Cancer Epidemiol Biomarkers Prev 3(1):67-71

51. Vasioukhin V et al (1994) Point mutations of the N-ras gene in the blood plasma DNA of patients with myelodysplastic syndrome or acute myelogenous leukaemia. $\mathrm{Br} \mathrm{J}$ Haematol 86(4):774-779

52. Kohler C et al (2009) Levels of plasma circulating cell free nuclear and mitochondrial DNA as potential biomarkers for breast tumors. Mol Cancer 8:105

53. Divella R et al (2009) Circulating hTERT DNA in early breast cancer. Anticancer Res 29(7):2845-2849

54. Van der Auwera I et al (2009) The presence of circulating total DNA and methylated genes is associated with circulating tumour cells in blood from breast cancer patients. Br J Cancer 100(8):1277-1286

55. van der Drift $\mathrm{M}$ et al (2010) Circulating DNA is a non-invasive prognostic factor for survival in non-small cell lung cancer. Lung Cancer 68(2):283-287

56. Yoon K et al (2009) Comparison of circulating plasma DNA levels between lung cancer patients and healthy controls. J Mol Diagn 11(3):182-185

57. Yen L et al (2009) Detection of KRAS oncogene in peripheral blood as a predictor of the response to cetuximab plus chemotherapy in patients with metastatic colorectal cancer. Clin Cancer Res 15(13):4508-4513

58. Ellinger $\mathrm{J}$ et al (2009) CpG island hypermethylation of cell-free circulating serum DNA in patients with testicular cancer. J Urol 182(1):324-329

59. Board R et al (2009) Detection of BRAF mutations in the tumour and serum of patients enrolled in the AZD6244 (ARRY142886) advanced melanoma phase II study. Br J Cancer 101(10):1724-1730 
60. Chuang $\mathrm{T}$ et al (2010) Detectable BRAF mutation in serum DNA samples from patients with papillary thyroid carcinomas. Head Neck 32(2):229-234

61. Schwarzenbach $\mathrm{H}$ et al (2009) Comparative evaluation of cellfree tumor DNA in blood and disseminated tumor cells in bone marrow of patients with primary breast cancer. Breast Cancer Res 11(5):R71

62. Marrakchi R et al (2008) Detection of cytokeratin 19 mRNA and CYFRA 21-1 (cytokeratin 19 fragments) in blood of Tunisian women with breast cancer. Int J Biol Markers 23(4):238-243

63. García V et al (2008) Free circulating mRNA in plasma from breast cancer patients and clinical outcome. Cancer Lett 263(2):312-320

64. O'Driscoll L et al (2008) Feasibility and relevance of global expression profiling of gene transcripts in serum from breast cancer patients using whole genome microarrays and quantitative RT-PCR. Cancer Genomics Proteomics 5(2):94-104

65. Terrin L et al (2008) Relationship between tumor and plasma levels of hTERT mRNA in patients with colorectal cancer: implications for monitoring of neoplastic disease. Clin Cancer Res 14(22):7444-7451

66. Vrieling A et al (2009) Expression of insulin-like growth factor system components in colorectal tissue and its relation with serum IGF levels. Growth Horm IGF Res 19(2):126-135

67. Fleischhacker M, Schmidt B (2007) Circulating nucleic acids (CNAs) and cancer-a survey. Biochim Biophys Acta 1775(1):181-232

68. Rieber M, Bacalao J (1974) An "external" RNA removable from mammalian cells by mild proteolysis. Proc Natl Acad Sci USA 71(12):4960-4964

69. Laktionov PP et al (2004) Cell-surface-bound nucleic acids: free and cell-surface-bound nucleic acids in blood of healthy donors and breast cancer patients. Ann NY Acad Sci 1022:221-227

70. Chelobanov BP et al (2004) Isolation of nucleic acid binding proteins: an approach for isolation of cell surface, nucleic acid binding proteins. Ann NY Acad Sci 1022:239-243

71. Wieczorek AJ, Rhyner K (1989) A gene probe test for serum RNA proteolipid in neoplasia. Schweiz Med Wochenschr 119(39):1342-1343

72. Wieczorek AJ et al (1985) Isolation and characterization of an RNA-proteolipid complex associated with the malignant state in humans. Proc Natl Acad Sci USA 82(10):3455-3459

73. Hasselmann DO et al (2001) Extracellular tyrosinase mRNA within apoptotic bodies is protected from degradation in human serum. Clin Chem 47(8):1488-1489

74. Stroun M et al (1978) Presence of RNA in the nucleoprotein complex spontaneously released by human lymphocytes and frog auricles in culture. Cancer Res 38(10):3546-3554

75. Jachertz D et al (1979) Information carried by the DNA released by antigen-stimulated lymphocytes. Immunology 37(4):753-763

76. Anker P et al (1999) Detection of circulating tumour DNA in the blood (plasma/serum) of cancer patients. Cancer Metastasis Rev 18(1):65-73

77. Jahr JS et al (2001) A novel approach to measuring circulating blood volume: the use of a hemoglobin-based oxygen carrier in a rabbit model. Anesth Analg 92(3):609-614

78. Skog J et al (2008) Glioblastoma microvesicles transport RNA and proteins that promote tumour growth and provide diagnostic biomarkers. Nat Cell Biol 10(12):1470-1476

79. Wright LC et al (1986) A proteolipid in cancer cells is the origin of their high-resolution NMR spectrum. FEBS Lett 203(2): 164-168

80. Rosi A et al (1988) RNA-lipid complexes released from the plasma membrane of human colon carcinoma cells. Cancer Lett 39(2):153-160
81. Ceccarini M et al (1989) Biochemical and NMR studies on structure and release conditions of RNA-containing vesicles shed by human colon adenocarcinoma cells. Int $\mathrm{J}$ Cancer 44(4):714-721

82. Ratajczak J et al (2006) Membrane-derived microvesicles: important and underappreciated mediators of cell-to-cell communication. Leukemia 20(9):1487-1495

83. Graner MW et al (2009) Proteomic and immunologic analyses of brain tumor exosomes. FASEB J 23(5):1541-1557

84. Thery $\mathrm{C}$ et al (2002) Exosomes: composition, biogenesis and function. Nat Rev Immunol 2(8):569-579

85. Keller S et al (2006) Exosomes: from biogenesis and secretion to biological function. Immunol Lett 107(2):102-108

86. Calzolari A et al (2006) TfR2 localizes in lipid raft domains and is released in exosomes to activate signal transduction along the MAPK pathway. J Cell Sci 119(Pt 21):4486-4498

87. Valadi $\mathrm{H}$ et al (2007) Exosome-mediated transfer of mRNAs and microRNAs is a novel mechanism of genetic exchange between cells. Nat Cell Biol 9(6):654-659

88. Safaei R et al (2005) Abnormal lysosomal trafficking and enhanced exosomal export of cisplatin in drug-resistant human ovarian carcinoma cells. Mol Cancer Ther 4(10):1595-1604

89. Chaput $\mathrm{N}$ et al (2005) The potential of exosomes in immunotherapy. Expert Opin Biol Ther 5(6):737-747

90. Liu $\mathrm{C}$ et al (2006) Murine mammary carcinoma exosomes promote tumor growth by suppression of NK cell function. J Immunol 176(3):1375-1385

91. Ginestra A et al (1998) The amount and proteolytic content of vesicles shed by human cancer cell lines correlates with their in vitro invasiveness. Anticancer Res 18(5A):3433-3437

92. Clayton A et al (2007) Human tumor-derived exosomes selectively impair lymphocyte responses to interleukin-2. Cancer Res 67(15):7458-7466

93. Ng EK et al (2002) Presence of filterable and nonfilterable mRNA in the plasma of cancer patients and healthy individuals. Clin Chem 48(8):1212-1217

94. O'Driscoll L (2007) Extracellular nucleic acids and their potential as diagnostic, prognostic and predictive biomarkers. Anticancer Res 27(3):1257-1265

95. Rabinowits $\mathrm{G}$ et al (2009) Exosomal microRNA: a diagnostic marker for lung cancer. Clin Lung Cancer 10(1):42-46

96. Silva JM et al (2001) Detection of epithelial messenger RNA in the plasma of breast cancer patients is associated with poor prognosis tumor characteristics. Clin Cancer Res 7(9):28212825

97. Chen X et al (2000) Telomerase RNA as a detection marker in the serum of breast cancer patients. Clin Cancer Res 6(10):3823-3826

98. Silva $J$ et al (2007) Circulating Bmi-1 mRNA as a possible prognostic factor for advanced breast cancer patients. Breast Cancer Res 9(4):R55

99. Fuchs E, Weber K (1994) Intermediate filaments: structure, dynamics, function, and disease. Annu Rev Biochem 63:345382

100. Dohmoto K et al (2001) The role of caspase 3 in producing cytokeratin 19 fragment (CYFRA21-1) in human lung cancer cell lines. Int J Cancer 91(4):468-473

101. Pujol JL et al (1993) Serum fragment of cytokeratin subunit 19 measured by CYFRA 21-1 immunoradiometric assay as a marker of lung cancer. Cancer Res 53(1):61-66

102. Ravdin PM et al (2001) Computer program to assist in making decisions about adjuvant therapy for women with early breast cancer. J Clin Oncol 19(4):980-991

103. Stathopoulou A et al (2002) Molecular detection of cytokeratin19-positive cells in the peripheral blood of patients with 
operable breast cancer: evaluation of their prognostic significance. J Clin Oncol 20(16):3404-3412

104. Xenidis $N$ et al (2006) Predictive and prognostic value of peripheral blood cytokeratin-19 mRNA-positive cells detected by real-time polymerase chain reaction in node-negative breast cancer patients. J Clin Oncol 24(23):3756-3762

105. Daskalaki A et al (2009) Detection of cytokeratin-19 mRNApositive cells in the peripheral blood and bone marrow of patients with operable breast cancer. Br J Cancer 101(4):589-597

106. Xenidis N et al (2003) Peripheral blood circulating cytokeratin19 mRNA-positive cells after the completion of adjuvant chemotherapy in patients with operable breast cancer. Ann Oncol 14(6):849-855

107. Quintela-Fandino M et al (2006) Breast cancer-specific mRNA transcripts presence in peripheral blood after adjuvant chemotherapy predicts poor survival among high-risk breast cancer patients treated with high-dose chemotherapy with peripheral blood stem cell support. J Clin Oncol 24(22):3611-3618

108. Wiedswang $\mathrm{G}$ et al (2004) Isolated tumor cells in bone marrow three years after diagnosis in disease-free breast cancer patients predict unfavorable clinical outcome. Clin Cancer Res 10(16): $5342-5348$

109. Ignatiadis $M$ et al (2008) Circulating tumor cells in breast cancer. Curr Opin Obstet Gynecol 20(1):55-60

110. Alix-Panabieres C et al (2009) Full-length cytokeratin-19 is released by human tumor cells: a potential role in metastatic progression of breast cancer. Breast Cancer Res 11(3):R39

111. DePinho R (2000) The age of cancer. Nature 408(6809): 248-254

112. Dasí $\mathrm{F}$ et al (2006) Real-time quantification of human telomerase reverse transcriptase mRNA in the plasma of patients with prostate cancer. Ann NY Acad Sci 1075:204-210

113. Li H et al (2009) Relationship between the expression of hTERT and EYA4 mRNA in peripheral blood mononuclear cells with the progressive stages of carcinogenesis of the esophagus. J Exp Clin Cancer Res 28:145

114. Elder E et al (2003) KI-67 and hTERT expression can aid in the distinction between malignant and benign pheochromocytoma and paraganglioma. Mod Pathol 16(3):246-255

115. Shen $C$ et al (2009) The detection of circulating tumor cells of breast cancer patients by using multimarker (Survivin, hTERT and hMAM) quantitative real-time PCR. Clin Biochem 42(3): 194-200

116. Jacobs JJ, van Lohuizen M (2002) Polycomb repression: from cellular memory to cellular proliferation and cancer. Biochim Biophys Acta 1602(2):151-161

117. Raaphorst FM (2005) Deregulated expression of Polycombgroup oncogenes in human malignant lymphomas and epithelial tumors. Hum Mol Genet 14 Spec No 1, R93-R100

118. Song LB et al (2009) The polycomb group protein Bmi-1 represses the tumor suppressor PTEN and induces epithelialmesenchymal transition in human nasopharyngeal epithelial cells. J Clin Invest 119(12):3626-3636

119. Bachmann IM et al (2006) EZH2 expression is associated with high proliferation rate and aggressive tumor subgroups in cutaneous melanoma and cancers of the endometrium, prostate, and breast. J Clin Oncol 24(2):268-273

120. Kleer CG et al (2003) EZH2 is a marker of aggressive breast cancer and promotes neoplastic transformation of breast epithelial cells. Proc Natl Acad Sci USA 100(20):11606-11611

121. van Lohuizen $M$ et al (1991) Identification of cooperating oncogenes in $\mathrm{E}$ mu-myc transgenic mice by provirus tagging. Cell 65(5):737-752

122. Liu S et al (2006) Hedgehog signaling and Bmi-1 regulate selfrenewal of normal and malignant human mammary stem cells. Cancer Res 66(12):6063-6071
123. Kim JH et al (2004) Overexpression of Bmi-1 oncoprotein correlates with axillary lymph node metastases in invasive ductal breast cancer. Breast 13(5):383-388

124. Vonlanthen $\mathrm{S}$ et al (2001) The bmi-1 oncoprotein is differentially expressed in non-small cell lung cancer and correlates with INK4A-ARF locus expression. Br J Cancer 84(10):13721376

125. Kim JH et al (2004) The Bmi-1 oncoprotein is overexpressed in human colorectal cancer and correlates with the reduced p16INK4a/p14ARF proteins. Cancer Lett 203(2):217-224

126. Klug $\mathrm{J}$ et al (2000) Uteroglobin/Clara cell $10-\mathrm{kDa}$ family of proteins: nomenclature committee report. Ann NY Acad Sci 923:348-354

127. Ni J et al (2000) All human genes of the uteroglobin family are localized on chromosome 11q12.2 and form a dense cluster. Ann NY Acad Sci 923:25-42

128. Watson MA, Fleming TP (1994) Isolation of differentially expressed sequence tags from human breast cancer. Cancer Res 54(17):4598-4602

129. Watson MA et al (1998) Structure and transcriptional regulation of the human mammaglobin gene, a breast cancer associated member of the uteroglobin gene family localized to chromosome 11q13. Oncogene 16(6):817-824

130. Tassi RA et al (2008) Mammaglobin B expression in human endometrial cancer. Int J Gynecol Cancer 18(5):1090-1096

131. Watson MA, Fleming TP (1996) Mammaglobin, a mammaryspecific member of the uteroglobin gene family, is overexpressed in human breast cancer. Cancer Res 56(4):860-865

132. Zafrakas $\mathrm{M}$ et al (2006) Expression analysis of mammaglobin A (SCGB2A2) and lipophilin B (SCGB1D2) in more than 300 human tumors and matching normal tissues reveals their coexpression in gynecologic malignancies. BMC Cancer 6:88

133. Nunez-Villar MJ et al (2003) Elevated mammaglobin (h-MAM) expression in breast cancer is associated with clinical and biological features defining a less aggressive tumour phenotype. Breast Cancer Res 5(3):R65-R70

134. Fleming TP, Watson MA (2000) Mammaglobin, a breast-specific gene, and its utility as a marker for breast cancer. Ann NY Acad Sci 923:78-89

135. Fanger GR et al (2002) Detection of mammaglobin in the sera of patients with breast cancer. Tumour Biol 23(4):212-221

136. Zach O et al (1999) Detection of circulating mammary carcinoma cells in the peripheral blood of breast cancer patients via a nested reverse transcriptase polymerase chain reaction assay for mammaglobin mRNA. J Clin Oncol 17(7):2015-2019

137. Cerveira N et al (2004) Highly sensitive detection of the MGB1 transcript (mammaglobin) in the peripheral blood of breast cancer patients. Int J Cancer 108(4):592-595

138. Marques AR et al (2009) Detection of human mammaglobin mRNA in serial peripheral blood samples from patients with non-metastatic breast cancer is not predictive of disease recurrence. Breast Cancer Res Treat 114(2):223-232

139. Mikhitarian K et al (2008) Detection of mammaglobin mRNA in peripheral blood is associated with high grade breast cancer: interim results of a prospective cohort study. BMC Cancer 8:55

140. Fu M et al (2004) Minireview: Cyclin D1: normal and abnormal functions. Endocrinology 145(12):5439-5447

141. Butt AJ et al (2005) Downstream targets of growth factor and oestrogen signalling and endocrine resistance: the potential roles of c-Myc, cyclin D1 and cyclin E. Endocr Relat Cancer 12(Suppl 1): S47-S59

142. Carney WP et al (2004) Monitoring the circulating levels of the HER2/neu oncoprotein in breast cancer. Clin Breast Cancer $5(2): 105-116$

143. Apostolaki S et al (2009) Detection of occult HER2 mRNApositive tumor cells in the peripheral blood of patients with 
operable breast cancer: evaluation of their prognostic relevance. Breast Cancer Res Treat 117(3):525-534

144. Ambros V (2004) The functions of animal microRNAs. Nature 431(7006):350-355

145. Hennessy E, O'Driscoll L (2008) Molecular medicine of microRNAs: structure, function and implications for diabetes. Expert Rev Mol Med 10:e24

146. Gartel A, Kandel E (2008) miRNAs: Little known mediators of oncogenesis. Semin Cancer Biol 18(2):103-110

147. Lawrie $\mathrm{C}$ et al (2008) Detection of elevated levels of tumourassociated microRNAs in serum of patients with diffuse large Bcell lymphoma. Br J Haematol 141(5):672-675

148. Mitchell P et al (2008) Circulating microRNAs as stable bloodbased markers for cancer detection. Proc Natl Acad Sci USA 105(30):10513-10518

149. Chen $X$ et al (2008) Characterization of microRNAs in serum: a novel class of biomarkers for diagnosis of cancer and other diseases. Cell Res 18(10):997-1006

150. Taylor D, Gercel-Taylor C (2008) MicroRNA signatures of tumor-derived exosomes as diagnostic biomarkers of ovarian cancer. Gynecol Oncol 110(1):13-21
151. Heneghan $\mathrm{H}$ et al (2010) Circulating microRNAs as novel minimally invasive biomarkers for breast cancer. Ann Surg 251(3):499-505

152. Duffy M (2005) Predictive markers in breast and other cancers: a review. Clin Chem 51(3):494-503

153. Gasparini G et al (2006) Is tailored therapy feasible in oncology? Crit Rev Oncol Hematol 57(1):79-101

154. Hayes D (2005) Prognostic and predictive factors for breast cancer: translating technology to oncology. J Clin Oncol 23(8):1596-1597

155. McShane L et al (2005) Reporting recommendations for tumor marker prognostic studies. J Clin Oncol 23(36):9067-9072

156. Barker AD et al (2009) I-SPY 2: an adaptive breast cancer trial design in the setting of neoadjuvant chemotherapy. Clin Pharmacol Ther 86(1):97-100

157. Pepe MS et al (2001) Phases of biomarker development for early detection of cancer. J Natl Cancer Inst 93(14):1054-1061 